\title{
Parallel Realization of Difference Schemes of Filtration Problem in a Multilayer System
}

\author{
Miron Pavluš ${ }^{1,2}$ and Edik Hayryan ${ }^{2}$ \\ 1 Technical University of Košice, Department of Mathematics, \\ Vysokoškolská 4, 04200 Košice, Slovakia \\ Miron.Pavlus@tuke.sk \\ 2 Joint Institute of Nuclear Research, Laboratory of Information Technology, \\ 141980 Dubna, Moscow Region, Russia \\ \{Pavlus, Ayrjan\}@cv.jinr.ru
}

\begin{abstract}
Early suggested difference schemes with splitting according to the physical processes are used and applied for a plane filtration problem in a multilayer system. Parallel algorithm connected with the solving of the filtration problem (one water-carrying layer on one processor) is constructed. Program realization on the multiprocessor system SPP2000 is discussed. Some results of sequential and parallel programs are compared with regard to the used time $M P I_{-}$Wtime() procedure.
\end{abstract}

\section{Introduction}

Modeling of the water filtration in practical applications of hydro-geology dynamics is based very often on the using of models of plane filtration [1], [2], [3], [4] with homogenization of filtration flows in the direction of the layer thickness. A typical situation occurs when horizontal water-carrying layers are alternated by the horizontal weakly permeable ground layers. Mathematical models of filtration in a multilayer system were constructed provided that a longitudinal flow of water takes place mainly in the water-carrying layers and cross flow of water takes place through separated layers (Mityaev-Girinski model [5]). These mathematical models can be valid by the homogenization theory [6] and represent a complex system.

Various methods [7] were used for numerical solution of the plane filtration problems. The simplest of them consists of using of common difference schemes with weights [8]. For example, the implicit scheme for a parabolic constrained system of equations for the piezometric head in each water-carrying layer can be used. After some suitable iteration process based on the idea of determining of piezometric head in each separate water-carrying layer can be applied. Difference schemes with splitting in space variables were constructed in [7]. But realization of such approach for a multilayer system even for one dimensional problems (the dependence only on one longitudinal variable) is constrained with using of three diagonal matrix inversion that, certainly, substantially complicates computational schemes. 
In this paper according to [9] we formulate a problem of the plane filtration in the multilayer systems and we use, suggested in [9], difference schemes with splitting that are free of mentioned inconvenience. The used difference schemes are splitted according to the physical processes when two processes - filtration along the water-carrying layers and flows between the layers are separated. Moreover, the schemes are additive and absolutely stable.

The formulation of the problem and difference schemes for its solution are presented in Section 2. Parallel algorithms for solution of the used difference schemes of filtration problems in multilayer systems are constructed in Section 3. In Section 3 we also suggest the most natural approach that consists of the solution when the filtration problem in each water-carrying layer is processed by one processor. Program realization of such approach is discussed for the multiprocessor system SPP2000 in Section 3 as well.

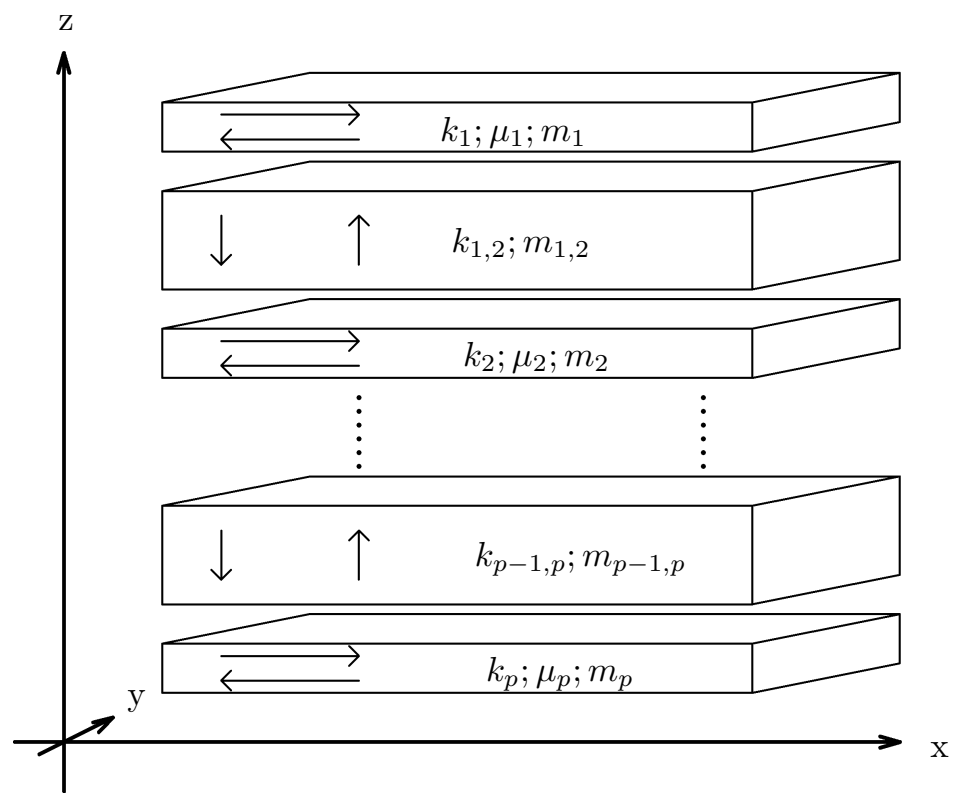

Fig. 1 A Multilayer System of Water-carrying and Weakly Permeable Layers

\section{Formulation of the Problem and Difference Schemes}

Let us consider, according to [9], the plane filtration in a multilayer system which consists (see Fig. 1) of $p$ water-carrying layers that are separated by weakly permeable layers. Let us suppose all layers are horizontal. We denote the hydrodynamical piezometric head $H_{\alpha}(x, y, t)$ in the point $(x, y)$ of the layer $\alpha, \alpha=$ $1,2, \ldots, p$ at the moment $t$. Let $k_{\alpha}=k_{\alpha}(x, y)$ be filtration coefficients, $m_{\alpha}-$ thickness of a water-carrying layer $\alpha, T_{\alpha}=k_{\alpha} m_{\alpha}$ - conductivity of layer and $\mu_{\alpha}=\mu_{\alpha}(x, y)$ - elastic capacity of layer. Correspondingly, $k_{\alpha, \beta}=k_{\alpha, \beta}(x, y), \beta=$ $\alpha+1, \alpha-1$ are filtration coefficients and $m_{\alpha, \beta}$ - thickness of a weakly permeable 
layer, $\chi_{\alpha, \beta}=k_{\alpha, \beta} / m_{\alpha, \beta}$ - coefficients of the cross flow. If the conditions of the cross flow $\left(k_{\alpha} / m_{\alpha}<<k_{\alpha, \beta} / m_{\alpha, \beta}\right)$ hold then we use for description of filtration in each water-carrying layer $\alpha, \alpha=2,3, \ldots, p-1$ the following equation

$$
\begin{gathered}
\mu_{\alpha} \frac{\partial H_{\alpha}}{\partial t}=\frac{\partial}{\partial x} T_{\alpha} \frac{\partial H_{\alpha}}{\partial x}+\frac{\partial}{\partial y} T_{\alpha} \frac{\partial H_{\alpha}}{\partial y}+ \\
+\chi_{\alpha, \alpha+1}\left(H_{\alpha+1}-H_{\alpha}\right)+\chi_{\alpha, \alpha-1}\left(H_{\alpha-1}-H_{\alpha}\right)+f_{\alpha}(x, y, t),
\end{gathered}
$$

where $f_{\alpha}(x, y, t)$ is a water source and/or a water sink. We have for lower and upper layers

$$
\begin{gathered}
\mu_{1} \frac{\partial H_{1}}{\partial t}=\frac{\partial}{\partial x} T_{1} \frac{\partial H_{1}}{\partial x}+\frac{\partial}{\partial y} T_{1} \frac{\partial H_{1}}{\partial y}+ \\
+\chi_{1,2}\left(H_{2}-H_{1}\right)-\chi_{1,0} H_{1}+f_{1}(x, y, t), \\
\mu_{p} \frac{\partial H_{p}}{\partial t}=\frac{\partial}{\partial x} T_{p} \frac{\partial H_{p}}{\partial x}+\frac{\partial}{\partial y} T_{p} \frac{\partial H_{p}}{\partial y}+ \\
+\chi_{p, p-1}\left(H_{p-1}-H_{p}\right)-\chi_{p, p+1} H_{p}+f_{p}(x, y, t),
\end{gathered}
$$

Equations (2), (3) for $\chi_{1,0}=0, \chi_{p, p+1}=0$ correspond to assumption that upper and lower layers are bounded by waterproof. Due to the mass conservation law we have in $(1)-(3)$

$$
\chi_{\alpha, \alpha+1}=\chi_{\alpha+1, \alpha}, \quad \alpha=1,2, \ldots, p-1 .
$$

The equations system (1) - (3) is considered on a calculation domain $D$. For simplicity we suppose the domain $D$ is a rectangle

$$
D=\{(x, y) \quad \mid \quad 0<x<a, \quad 0<y<b\} .
$$

We take boundary conditions in the simplest form

$$
H_{\alpha}(x, y, t)=0, \quad(x, y) \in \partial D, \quad \alpha=1,2, \ldots, p .
$$

Finally, initial conditions must be written

$$
H_{\alpha}(x, y, 0)=H_{\alpha}^{0}(x, y), \quad(x, y) \in D, \quad \alpha=1,2, \ldots, p .
$$

We rewrite the imposed initial boundary value problem (1) - (6) in the brief form. Let us introduce some denotations. Let the vector

$$
U=U(x, y, t)=\left\{H_{1}, H_{2}, \ldots, H_{p}\right\}
$$

be a solution of the problem (1) - (6), and

$$
F=F(x, y, t)=\left\{f_{1}, f_{2}, \ldots, f_{p}\right\} .
$$

Let us define the diagonal matrices $M$ and $\theta$

$$
M=\left\{\mu_{\alpha}(x, y) \delta_{\alpha, \beta}\right\}, \quad \alpha, \beta=1,2, \ldots, p,
$$




$$
\theta=\left\{T_{\alpha}(x, y) \delta_{\alpha, \beta}\right\}, \quad \alpha, \beta=1,2, \ldots, p,
$$

where $\delta_{\alpha, \beta}$ be the Kronecker's symbol. Now, let $K$ is a three diagonal matrix that corresponds to cross flow through separating layers

$$
K=\left\{\kappa_{\alpha, \beta}\right\}, \quad \alpha, \beta=1,2, \ldots, p,
$$

in which connection

$$
\kappa_{\alpha, \alpha-1}=-\chi_{\alpha, \alpha-1}, \quad \kappa_{\alpha, \alpha+1}=-\chi_{\alpha, \alpha+1}, \quad \kappa_{\alpha, \alpha}=\chi_{\alpha, \alpha-1}+\chi_{\alpha, \alpha+1} .
$$

Finally, we define

$$
L U=-\frac{\partial}{\partial x} \theta \frac{\partial U}{\partial x}-\frac{\partial}{\partial y} \theta \frac{\partial U}{\partial y} .
$$

Taking into account $(7)-(10)$ we write the system of equations $(1)-(3)$ in the form

$$
M \frac{\partial U}{\partial t}+L U+K U=F(x, y, t), \quad(x, y) \in D .
$$

Using analogical denotations the boundary and initial conditions (5), (6) have the following form

$$
\begin{gathered}
U(x, y, t)=0, \quad(x, y) \in \partial D, \\
U(x, y, 0)=U^{0}(x, y), \quad(x, y) \in D .
\end{gathered}
$$

Next, we introduce difference schemes with weights for solution of the brief formulated problem (11) - (13). Let us introduce the equidistant net

$$
\omega_{\tau}=\left\{t \mid t=t_{n}=n \tau, \quad n=0,1, \ldots\right\}
$$

with respect to time variable the time step of which is $\tau>0$. We also examine the approximated equations $(11)-(13)$ with respect to the space variables discretization. We introduce an equidistant rectangle net in the rectangular domain $D$ with discretizing parameters $h_{x}$ and $h_{y}$

$$
\begin{gathered}
\bar{\omega}_{h}=\omega_{h}+\gamma_{h}=\left\{(x, y) \mid(x, y)=\left(x_{i}, y_{j}\right),\right. \\
x_{i}=i h_{x}, i=0,1, \ldots, N_{x}, \quad y_{j}=j h_{y}, j=0,1, \ldots, N_{y}, \\
\left.N_{x} h_{x}=a, N_{y} h_{y}=b\right\},
\end{gathered}
$$

where $\omega_{h}$ means the set of inner nodes and $\gamma_{h}$ means the set of boundary nodes of defined net. We denote $V^{n}$ the approximate solution of the problem (11) - (13) at the moment $t_{n}$. Note, the constructed operators $M, L$ and $K$ are constant (do not depend on $n$ ). Now, the used difference schemes are the following

$$
\begin{gathered}
M \frac{V^{n+1 / 4}-V^{n}}{\tau}+L_{h x}\left[\sigma_{1} V^{n+1 / 4}+\left(1-\sigma_{1}\right) V^{n}\right]=F^{n}, \\
M \frac{V^{n+1 / 2}-V^{n+1 / 4}}{\tau}+L_{h y}\left[\sigma_{1} V^{n+1 / 2}+\left(1-\sigma_{1}\right) V^{n+1 / 4}\right]=F^{n},
\end{gathered}
$$




$$
\begin{gathered}
M \frac{V^{n+3 / 4}-V^{n+1 / 2}}{\tau}+K^{+}\left[\sigma_{2} V^{n+3 / 4}+\left(1-\sigma_{2}\right) V^{n+1 / 2}\right]=F^{n} \\
M \frac{V^{n+1}-V^{n+3 / 4}}{\tau}+K^{-}\left[\sigma_{2} V^{n+1}+\left(1-\sigma_{2}\right) V^{n+3 / 4}\right]=F^{n}
\end{gathered}
$$

where

$$
\begin{gathered}
L_{h}=L_{h x}+L_{h y}, \quad L_{h x} V_{h}=-\left(\theta_{1} V_{h \bar{x}}\right)_{x}, \quad L_{h y} V_{h}=-\left(\theta_{2} V_{h \bar{y}}\right)_{y}, \\
K=K^{+}+K^{-}, \quad\left(K^{+}\right)^{*}=K^{-}
\end{gathered}
$$

i.e. the three diagonal matrix $K$ is splitted to lower and upper triangular matrices. Generally speaking the existence of the inverse operator $K^{-1}$ doesn't take place in our case (see equations (1)-(3) and condition (4)). We observe a more pleasant situation when upper and lower layers are not bounded by waterproof but also by weakly permeable layers i.e. $\chi_{1,0}$ and $\chi_{p, p+1}$ are positive. The schemes $(14)-(17)$ were constructed in [9] and are additive. It was proven in [9] that if $F^{n}=0, \sigma_{1} \geq 0.5$ and $\sigma_{2}=1$ then the schemes are stable with regard to the initial condition $V^{0}=U^{0}$. The stability according to the right hand side $F^{n}$ can be also established. Moreover, the schemes are economical (the number of arithmetical operations related to one node when moving to a next time layer doesn't depend on the general number of nodes). The schemes possess the following important property. The transfer of water in the water-carrying layers are described by the first two time quarter-steps (see (14), (15)) and by the last two time quarter-steps (see (16), (17)) the transfer between weakly permeable layers are described. In this context the schemes (14) - (17) of sum approximation can be treated as schemes of splitting according to the physical processes.

\section{Parallel Realization of Difference Schemes}

Let us consider the difference scheme (14) - (17) of splitting of the problem according to the physical processes. On the first time half-step $((14),(15))$ we consider the water transfer in the horizontal water-carrying layers and on the second time half-step $((16),(17))$ we consider the flow of the water in the vertical direction through the weakly permeable layers. The equations (14), (15) represent systems of linear algebraic equations with symmetric three diagonal matrices on the each water-carrying layer $\alpha$. Equations (16), (17) tie together unknowns on the water-carrying layers and represent systems of linear algebraic equations with upper and lower triangle two diagonal matrices, respectively.

The most natural parallel realization of the difference scheme consists of the solution of the filtration problem on one processor for each water-carrying layer. In this case one time step of the difference scheme can be realized by the following algorithm.

1. Each fixed processor $\alpha$ has values of the piezometric head $v_{\alpha}^{n}, \alpha=1,2, \ldots, p$ on the net $\omega_{h}$ on the water-carrying layer $\alpha$ e.i. processor $\alpha$ contains $\alpha$-th component of the vector $V^{n}$. 
2. According to (14) we use $N_{y}-1$ of the Gauss elimination in the direction $x$ on each water-carrying layer. As a result we receive values $V^{n+1 / 4}$.

3. According to (15) we use $N_{x}-1$ of the Gauss elimination in the direction $y$ on each water-carrying layer. As a result we receive values $V^{n+1 / 2}$.

In cases 2 . and 3 . the Gauss eliminations are realized in parallel, need no data exchange and represent well known ADI method [10], [11].

4. We solve the system (16) with the upper triangle matrix from the bottom to the top (from the layer $p$ to the first layer) for all nodes $\omega_{h}$. As a result we receive values $V^{n+3 / 4}$.

5. We solve the system (17) with the lower triangle matrix from the top to the bottom (from the first layer to the layer $p$ ) for all nodes $\omega_{h}$. As a result we receive values $V^{n+1}$.

However, an effective parallel realization of the steps 4 . and 5 . requires data exchange between processors. For this reason we divide the net $\omega_{h}$ on $p$ equal parts choosing the value $N_{x}$ in the form $N_{x}=p k_{x}+1$ and we introduce auxiliary nets (see Fig. 2)

$$
\begin{gathered}
\omega_{\alpha}=\left\{\left(x_{i}, y_{j}\right) \mid x_{i}=i . h_{x}, i=(\alpha-1) k_{x}+1,(\alpha-1) k_{x}+2, \ldots, \alpha k_{x}\right. \\
\left.y_{j}=j . h_{y}, j=1,2, \ldots, N_{y}-1\right\}
\end{gathered}
$$

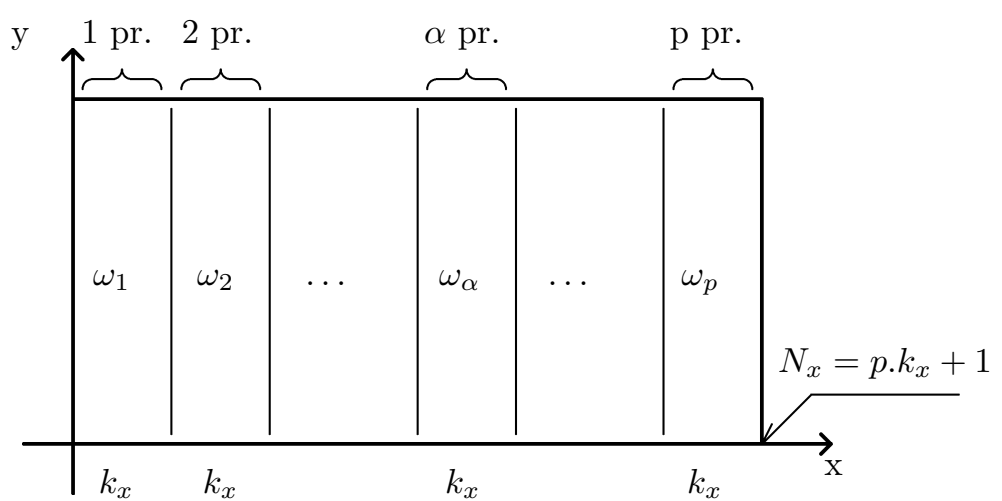

Fig. 2 Distribution of the Net Points between $\mathbf{p}$ Processors

We prescribe to processor $\alpha$ the processing of unknowns on the net $\omega_{\alpha}$. The processor $\alpha$ before the 4th step

- receives values $v_{1}^{n+1 / 2}, \ldots, v_{\alpha-1}^{n+1 / 2}, v_{\alpha+1}^{n+1 / 2}, \ldots, v_{p}^{n+1 / 2}$, on the nets $\omega_{\alpha}$ from processors $1, \ldots, \alpha-1, \alpha+1, \ldots, p$ and puts them in stead of unknowns $v_{\alpha}^{n+1 / 2}$ on the nets $\omega_{\beta}, \beta=1, \ldots, \alpha-1, \alpha+1, \ldots, p$

- transmits values $v_{\alpha}^{n+1 / 2}$ from nets $\omega_{\beta}, \beta=1, \ldots, \alpha-1, \alpha+1, \ldots, p$ to processor $\beta$. 
For finishing one time step of difference scheme it remains "to return" obtained values $V^{n+1}$ to correspondent processors.

Two codes for one and for $p$ processors $p=3,4,5,6$ were written for multiprocessor system SPP2000 using FORTRAN'77 with application of MPI (Message Passing Interface) [12]. The code for one processor (sequential program) processes sequentially $p$ water-carrying layers. The following coefficients of equations, initial and boundary conditions, parameters of difference scheme were chosen

$$
\begin{gathered}
a=b=1, \quad \tau=0.1, \quad \sigma_{1}=0.5, \quad \sigma_{2}=1, \\
f_{\alpha}(x, y, t)=0, \quad V_{\alpha}^{0}(x, y)=\alpha x(a-x) y(b-y) / p, \quad \mu_{\alpha}(x, y)=\alpha / p, \\
\theta_{1 \alpha}(x, y)=\alpha\left(x-\frac{h_{x}}{2}\right) y / p, \quad \theta_{2 \alpha}(x, y)=\alpha x\left(y-\frac{h_{y}}{2}\right) / p, \quad \chi_{\alpha, \alpha-1}=\chi_{\alpha, \alpha+1}=5, \\
\alpha=1,2, \ldots, p .
\end{gathered}
$$

Calculations were carried out for three following nets

$$
\left(N_{x}, N_{y}\right)=\{(151,100) ;(301,200) ;(601,400)\},
$$

where $p$ and $k_{x}$ in the representation $N_{x}=p k_{x}+1$ were chosen according to Table 1 .

Table 1. $p$ and $k_{x}$ parameters in representation $N_{x}=p k_{x}+1$

\begin{tabular}{llll}
\hline$p$ & $k_{x 1}$ & $k_{x 2}$ & $k_{x 3}$ \\
\hline 3 & 50 & 100 & 200 \\
4 & 38 & 75 & 150 \\
5 & 30 & 60 & 120 \\
6 & 25 & 50 & 100 \\
\hline
\end{tabular}

The Fig.3 presents the dependence of work time of processors on the number of layers. A "plus" sign denotes the mean time of a processor work if the parallel code was applied. A "minus" sign denotes time of a single processor for comparison with the sequential code. Both cases correspond to $p$ water-carrying layers $p=3,4,5,6$. We remind that in parallel code the number $p$ also denotes the number of used processors. The MPI_Wtime() procedure for time counting out was used. The values of time are in seconds. The Fig. 3 shows that calculation time of parallel code inessentially increases when the number $p$ of water-carrying layers increases. This is a good evidence of parallelism of algorithm. 

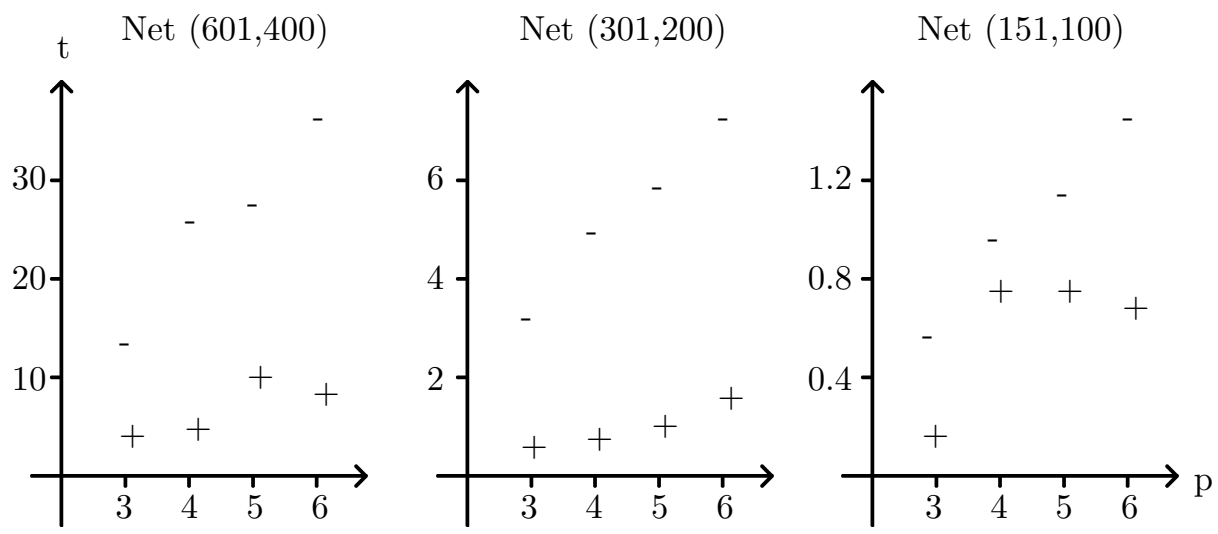

Fig. 3 The Dependence of Processors Work Time on the Number of Layers respectively on the Number of Processors

\section{Concluding Remarks}

At present the existence theorem was not proved neither for exact nor for weak solution of the formulated problem (11) - (13) but the proof of existence follows from the estimations given in [9].

Present paper deals with only parallel realization of the specific difference scheme. The used ADI method (equations (14)-(15)) can be replaced by other methods like FEM or a suitable modification of relaxation method. That means the suggested parllelism does not depend on the Gauss eliminations in steps 2. and 3. of parallel realization of difference schemes. A main advantage of the scheme consists of the following. It reflects physical processes - a longitudinal flow of water takes place mainly in the water-carrying layers and cross flow of water takes place through separated layers. The parallel realization conserves this property and can be used for modeling, for example, a water or naphtha movement in the multilayer system. This realization speeds up the calculation process and opens a new way for the multilayer systems modeling.

\section{Acknowledgement}

Authors wish to express thanks to Russian Fond of Fundamental Research, Grant 99-01-01101, with the support of which this paper was executed. Authors also thank Mr. Sapozhnikov A.P. and Mrs. Sapozhnikova T.F. for help with SPP2000. We thank Austrian Center in Košice for the conference ICCS2002 presentation support. We also thank anonymous reviewers for their helpful comments.

\section{References}

1. Fried, J.J.: Groundwater Pollution. Elsevier, New York (1975) 
2. Kinzelbach, W.: Groundwater Modeling. Elsevier, New York (1986)

3. Gavich, I.K.: Hydrogeodynamics. (in Russian), Nauka, Moscow (1988)

4. Polubarinova-Kochina, P.Ya.: Theory of Groundwater Movement. (in Russian), Nauka, Moscow (1977)

5. Mityaev, A.N.: Pushing Complex of Underground Waters and Wells. (in Russian), Izv. AN SSSR Otdel Techn. Nauk, 9, (1947) 1069-1088

6. Sanchez-Palencia, E.: Non-Homogeneous Medium and Vibration Theory. Lecture Notes in Physics 127, Springer-Verlag, New York (1980)

7. Lomakin, E.A., Mironenko, V.A., Shestakov, V.M.: Numerical Modeling of Geofiltration. (in Russian), Nedra, Moscow (1988)

8. Samarskij, A.A.: Theory of Difference Schemes. (in Russian), Nauka, Moscow (1983)

9. Hayryan, E.A., Vabishchevich, P.N., Pavluš, M., Fedorov, A.V.: Additive Difference Schemes for Filtration Problems in Multilayer Systems. (in Russian), preprint of the Joint Institute of Nuclear Research P11-2000-51, Dubna (2000)

10. Peaceman, D.W., Rachford H.H.: The Numerical Solution of Parabolic and Elliptic Differential Equation, J.Soc.Indus.Appl.Math. 3, (1955) 28-42

11. Young D.M.: Iterative Solutions of Large Linear Systems, Academic Press, New York (1971)

12. Snir, M., Otto, S.W., Huss-Lederman, S., Walker, D.W., Dongarra, J.: MPI. The Complete Reference. The MIT Press, Massachusetts Institute of Technology, Cambridge (USA) (1997) 